\title{
Reply to the correspondence letter by M.D. Majewska: Krakow's children and cognitive function: can the study by Jedrychowski et al. show us the bigger picture?
}

\author{
Wieslaw Jedrychowski
}

Received: 11 October 2011 / Accepted: 11 October 2011 / Published online: 21 October 2011

(C) Springer-Verlag 2011

\section{Dear Editor,}

M.D. Majewska and J. Dorea in their letter raised important issues related to significant confounders potentially implicated in the assessment of cognitive development of children in the context of breastfeeding practices. The authors mention that one of important confounders, which has not been considered in any of the publications, was ethyl mercury exposure during pregnancy and during the postnatal period through thimerosal-containing vaccines (TCVs). Indeed, TCVs were used in Poland during the time of the cohort formation. We have collected data on TCVs, but our analysis did not confirm a significant effect of TCV on cognitive development of children. However, its effect on psychomotor development appeared to be significant, although very weakly so. The effect of TVCs on psychomotor development of children is to be presented in a separate paper, which is currently in the preparatory stage (Mrozek et al.). The latter data have to be discussed in the light of experimental findings reviewed by Dr. Dorea; infant monkeys having a delay in reflexes (root, snout, and suck) may have problems with successful breastfeeding, though in our study the duration of exclusive breastfeeding was not associated with the psychomotor development score of infants.

We note that neither fish consumption in pregnancy (questionnaire data) nor prenatal exposure to lead (cord blood lead) or postnatal lead exposure (lead measured in urine of 5-year-olds) affected the beneficial effect attributed to long exclusive breastfeeding. Although in the study anti-RhoD immune globulins administered in pregnancy were not accounted for, we do not believe that it could significantly have biased the results, as the estimates of exposure resulting from this procedure in terms of the number of pregnant women treated and doses received are very low (estimates based on hospital medical records). Finally, we agree that the Krakow birth cohort study offers an excellent opportunity to perform comprehensive analysis of potential factors responsible for the neurodevelopment of children and an integrated approach is planned after completing the follow-up.

Sincerely yours, Wieslaw Jedrychowski, M.D., PhD
W. Jedrychowski $(\bowtie)$

Jagiellonian University,

Krakow, Poland

e-mail: myjedryc@cyf-kr.edu.pl 